\title{
Oligopólios e publicação científica: a busca por impacto na América Latina
}

\author{
Oligopolies and scientific publication: the \\ search for impact in Latin America
}

\author{
Patricia da Silva NEUBERT1 (iD) 0000-0002-8909-1898 \\ Rosângela Schwarz RODRIGUES² (D) 0000-0002-9639-6390
}

\begin{abstract}
Resumo
Este artigo investiga os editores da produção científica latino-americana. Os dados coletados na Web of Science entre os anos de 2014 a 2016 mostram 251.312 artigos em 11.965 periódicos, publicados majoritariamente por editoras comerciais (56,48\%), dentre as quais se destacam Elsevier, Springer, Wiley, Taylor \& Francis e Sage Group. Essas instituições concentram o maior volume de títulos e artigos em Ciências da Vida \& Biomedicina, Ciências Físicas e em Tecnologia. Nas Ciências Sociais e Artes \& Humanidades sobressaem-se os editores universitários da região. Discute-se o investimento para publicar ao estimar os valores das taxas de publicação cobradas pelos títulos com maior número de artigos. A conclusão aponta a relevância da editoria universitária regional para publicação em títulos mainstream nos campos relegados pela Web of Science e seu papel na promoção do acesso aberto pela via platina. Por fim, aponta-se o papel exercido pelos grandes editores comerciais no acompanhamento de demandas de abertura científica, sem perda de mercado.
\end{abstract}

Palavras-chave: América Latina. Bases de dados. Editores. Produção científica. Publicações periódicas.

\begin{abstract}
This article investigates the publishers of Latin American scientific production. The data collected on the Web of Science in the years of 2014 to 2016 show 251,312 articles in 11,965 journals, mostly by commercial publishers (56.48\%), among which Elsevier, Springer, Wiley, Taylor \& Francis, and the Sage Group stand out. These institutions concentrate the largest volume of titles and articles in the Life Sciences \& Biomedicine, Physical Sciences, and Technology. In the Social Sciences and in Arts \& Humanities, university publishers in the region stand out. The investment in publication is discussed when estimating the values of publication fees in titles with the largest number of articles. The conclusion points to the relevance of regional university publishing for publication in mainstream titles in fields relegated by the Web of Science and its role in promoting open access via platinum. It points out the strategies of large commercial publishers to keep up with demands of scientific openness without losing the market.
\end{abstract}

Keywords: Latin America. Databases. Publishers. Scientific production. Periodicals publication.

\footnotetext{
1 Universidade Federal de Santa Catarina, Programa de Pós-Graduação em Ciência da Informação. Campus Universitário Reitor João David Ferreira Lima, s/n., Trindade, 88040-900, Florianópolis, SC, Brasil. Correspondência para/Correspondence to: P. S. NEUBERT. E-mail: <patyneubert@hotmail.com>.

2 Universidade Federal de Santa Catarina, Departamento de Ciência da Informação, Programa de Pós-Graduação em Ciência da Informação. Florianópolis, SC, Brasil.

Apoio/Support: Coordenação de Aperfeiçoamento de Pessoal de Nível Superior (Código de Financiamento 001).

Artigo elaborado a partir da tese de P. S. NEUBERT, intitulada "Publicações científicas em títulos mainstream: a situação latino-americana". Universidade Federal de Santa Catarina, 2020.

Recebido em 20 de outubro de 2020, reapresentado em 5 de fevereiro de 2021 e aprovado em 8 de abril de 2021.
}

Como citar este artigo/How to cite this article

Neubert, P. S.; Rodrigues, R. S. Oligopólios e publicação cientíica: a busca por impacto na América Latina. Transinformação, v. 33, e200069, 2021. https:// doi.org/10.1590/2318-0889202133e200069 


\section{Introdução}

Até a Segunda Guerra Mundial a maioria dos periódicos havia sido criado por sociedades científicas. A identificação dos títulos de núcleo e a criação do Science Citation Index (SCI), acompanhada da publicação do Fator de Impacto (Fl), impulsionou a atuação de publishers comerciais no mercado de comunicação científica.

A passagem ao suporte digital e a alteração do modelo de negócio favoreceram a concentração da literatura científica em um grupo de empresas (Gingras, 2020; Lachenmayer, 2019; Lariviére; Hausten; Mongeo, 2015). As aquisições e fusões concentraram os títulos de núcleo em poucos publishers; mais de 50\% dos documentos da Web of Science (WoS) foram publicados por Elsevier, Wiley, Springer, Taylor \& Francis, Sage Group e American Chemical Society (Lariviére; Hausten; Mongeo, 2015). Esses números explicitam o oligopólio dos títulos de núcleo (Guédon, 2011), o que contribui para o aumento das margens de lucro (Aspesi et al., 2019; McGuigan; Russell, 2008; Monbiot, 2011) e para a manutenção da influência desses editores no sistema global de comunicação da Ciência.

Essa influência está alicerçada no domínio das bases de dados, que indexam os títulos segundo seus interesses - Scopus é propriedade do grupo Elsevier e a WoS é propriedade da Clarivate Analytics, anteriormente empresa do grupo Thomson Reuters e adquirida pelas investidoras Onex Corporation e Baring Private Equity Asia em 2016 (Elsevier, 2021; Thomson Reuters, 2016). Como são utilizados como indicadores de qualidade, esses indexadores são considerados essenciais para a avaliação de desempenho de pesquisadores e instituições.

Conforme publicam mais periódicos considerados núcleo da Ciência, e possuam seu copyright, adquirem controle sobre o sistema global de comunicação científica e exercem poder financeiro e político (Aspesi et al., 2019; Lachenmayer, 2019). Sua interferência nas temáticas publicadas, no acesso e preço dos periódicos, nas métricas e nos padrões técnicos e operacionais influencia globalmente a pauta da Ciência uma vez que projetos, linhas de pesquisa, financiamentos e status são redefinidos baseados no potencial de aceite e impacto das pesquisas.

Nesse sentido, o mercado editorial científico global é controlado por um pequeno grupo de editores, proprietários da maioria dos títulos de núcleo, atuantes em um mercado inelástico caracterizado pela ausência de concorrência e cujo oligopólio permite determinar o aumento das taxas de acesso ou publicação de acordo com seus interesses (Aspesi et al., 2019; Guédon, 2011; Lariviére; Haustein; Mongeo, 2015; McGuigan; Russell, 2008; Monbiot, 2011; Vessuri; Guédon; Cetto, 2014; Zepetnek; Jia, 2014). Com a expansão do Acesso Aberto, o oligopólio vem aderindo ao movimento por meio da cobrança de taxas para publicação em títulos híbridos ou dourados (Ellers; Crowter; Harvey, 2017). Dessa forma, os editores comerciais ampliam sua atuação, atendendo apelos dos diversos atores científicos pelo acesso livre e mantendo o domínio do mercado.

Na América Latina, região sem tradição editorial comercial, o desenvolvimento da web e os movimentos de abertura da Ciência proporcionaram um cenário profícuo ao surgimento de um modelo editorial alternativo. As universidades e as associações científicas se organizaram e são reconhecidas como os principais editores de periódicos científicos da região, publicando majoritariamente em Acesso Aberto usando a via platina e com edição e publicação custeadas pelo publisher (Repiso, 2019; Repiso; Orduña-Malea; Aguaded, 2019; Rodrigues; Abadal, 2014). Entretanto, ao longo do tempo tem permanecido a dificuldade de indexação desses títulos em bases de dados internacionais por sua limitada cobertura.

Nesse cenário, a publicação e o acesso aos periódicos de núcleo, atrelados às restrições financeiras, são dificultados em países de ciência periférica. As limitações associadas à indexação de periódicos latino-americanos resultam em uma representação distorcida da produção científica regional em estudos mundiais que consideram essas bases como fonte, nas quais se observa um movimento de evasão da produção científica dos países periféricos. Esse movimento contribui para a manutenção da cisão entre ciência de centro e de periferia com reflexos nos mecanismos de avaliação das produçães nacionais, se convertendo em uma barreira à criação e manutenção de periódicos nesses países e privilegiando a publicação em periódicos mainstream. 
Nesse contexto, a identificação dos publishers destino da produção científica da América Latina publicada em títulos de núcleo propicia um panorama acerca da representação do modelo adotado na região e dos padrões editoriais dos núcleos científicos. Nesse sentido, este artigo investiga a tipologia e a participação dos editores responsáveis pelos periódicos nos quais é publicada a produção científica latino-americana indexada na WoS.

\section{Procedimentos Metodológicos}

Na Coleção Principal da WoS, via Portal da Capes, foram obtidos os dados da produção latino-americana através da busca pelos países latino-americanos no campo de país (CU), por tipo de documento (article), por documentos produzidos no período de 2014-2016 e sem limitações de idiomas. A origem da produção científica, vinculada à filiação institucional dos autores, foi obtida pelo endereço adicionado na descrição da publicação. A estratégia de busca foi elaborada considerando as diferenças na grafia dos nomes dos países nos idiomas inglês, português e espanhol ${ }^{3}$ e a busca resultou em 251.312 artigos.

A classificação por campo do conhecimento foi realizada a partir do agrupamento da categorização de assuntos utilizada na WoS (subject category, campo WC) em cinco grandes campos: Arts \& Humanities, Life Sciences \& Biomedicine, Physical Sciences, Social Sciences e Technology. A classificação da tipologia editorial foi realizada a partir da informação disponibilizada pela base acerca da editoria dos títulos na qual os artigos foram publicados, informação registrada no campo PU. Os editores foram categorizados em Universidades, Associações, Comerciais, Fundações, Governos e Institutos. Quando a denominação informada na base impossibilitava a identificação da categoria institucional com clareza, recorreu-se à pesquisa no Google através da denominação informada ou do título e do International Standard Serial Number (ISSN) do periódico.

\section{Resultados e Discussão}

Os artigos foram publicados em 11.965 periódicos distribuídos em Editores Comerciais (56,48\%), Universidades (18,30\%) e Associações (19,25\%), mesma proporção observada na análise da editoria dos títulos: 66,67\%, $14,75 \%$ e 12,92\%, respectivamente.

Na WoS, os publishers comerciais são os maiores publicadores de periódicos, resultado refletido na produção indexada (Corera-Álvarez; Molina-Molina, 2016; Lariviére; Haustein; Mongeo, 2015). Entretanto, embora possuam maior volume de periódicos, o volume de artigos é aproximadamente 10\% menor que o número de títulos - fenômeno que se repete em quase todos os campos, exceto Tecnologia. O inverso ocorre com as universidades, que têm menor percentual de títulos em relação aos artigos - respectivamente 14,75\% e 18,30\%, exceto do campo de Ciências Físicas -, e nas associações, publicadoras de 12,92\% dos periódicos e 19,25\% dos artigos, exceto dos campos Tecnologia, Ciências Sociais e Artes \& Humanidades (Tabela 1).

\section{Publishers comerciais}

Publishers comerciais são maioria na publicação dos periódicos (Tabela 1). Sua presença é segmentada em subsidiárias e identificada pelo agrupamento dessas empresas em 408 grupos (responsáveis por 7.977 periódicos e 141.947 artigos). A maior parte dos títulos (7.294 - 91,44\%) e dos artigos (127.700 - 89,96\%) está concentrada em $10 \%$ dos grupos editoriais (40 empresas).

\footnotetext{
${ }^{3} \mathrm{CU}=$ (Argentina OR Bolivia OR Brazil OR Brasil OR Chile OR Colombia OR "Costa Rica"OR Cuba OR Ecuador OR Equador OR"El Salvador" OR Guatemala OR Haiti OR Honduras OR Mexico OR Mejico OR Nicaragua OR Panama OR Paraguay OR Paraguai OR Peru OR "Puerto Rico" OR "Porto Rico" OR "Republica Dominicana" OR "Dominican Republic" OR Uruguay OR Uruguai OR Venezuela).
} 
Tabela 1 - Artigos e periódicos por editoria e campo do conhecimento.

\begin{tabular}{|c|c|c|c|c|c|c|c|c|c|c|c|c|}
\hline \multirow{3}{*}{ Editoria } & \multirow{2}{*}{\multicolumn{2}{|c|}{$\begin{array}{c}\text { América } \\
\text { Latina } \\
N(\%) \\
\end{array}$}} & \multirow{2}{*}{\multicolumn{2}{|c|}{$\begin{array}{c}\text { Ciências da Vida } \\
\text { \& Biomedicina }\end{array}$}} & \multirow{2}{*}{\multicolumn{2}{|c|}{$\begin{array}{c}\text { Ciências } \\
\text { Físicas } \\
\mathrm{N}(\%)\end{array}$}} & \multicolumn{2}{|c|}{ Tecnologia } & \multicolumn{2}{|c|}{$\begin{array}{c}\text { Ciências } \\
\text { Sociais }\end{array}$} & \multirow{2}{*}{\multicolumn{2}{|c|}{$\begin{array}{c}\text { Artes \& } \\
\text { Humanidades }\end{array}$}} \\
\hline & & & & & & & & & & & & \\
\hline & A & $P$ & A & $P$ & A & $P$ & A & $P$ & A & $P$ & A & $P$ \\
\hline Comercial & $\begin{array}{l}141.947 \\
(56,48)\end{array}$ & $\begin{array}{l}7.977 \\
(66,67)\end{array}$ & $\begin{array}{l}87.218 \\
(60,93)\end{array}$ & $\begin{array}{c}4.443 \\
(74,85)\end{array}$ & $\begin{array}{l}34.478 \\
(59,85)\end{array}$ & $\begin{array}{l}1.417 \\
(67,00)\end{array}$ & $\begin{array}{l}34.314 \\
(69,64)\end{array}$ & $\begin{array}{l}1.540 \\
(66,67)\end{array}$ & $\begin{array}{c}8.039 \\
(33,71)\end{array}$ & $\begin{array}{l}1.646 \\
(62,35)\end{array}$ & $\begin{array}{c}874 \\
(11,48)\end{array}$ & $\begin{array}{c}299 \\
(37,10)\end{array}$ \\
\hline Universidade & $\begin{array}{l}45.994 \\
(18,30)\end{array}$ & $\begin{array}{c}1.765 \\
(14,75)\end{array}$ & $\begin{array}{l}21.392 \\
(14,95)\end{array}$ & $\begin{array}{c}465 \\
(7,83)\end{array}$ & $\begin{array}{l}3.909 \\
(6,79)\end{array}$ & $\begin{array}{c}178 \\
(8,42)\end{array}$ & $\begin{array}{l}4.034 \\
(8,19)\end{array}$ & $\begin{array}{c}176 \\
(7,62)\end{array}$ & $\begin{array}{l}13.018 \\
(54,59)\end{array}$ & $\begin{array}{c}704 \\
(26,67)\end{array}$ & $\begin{array}{c}5.614 \\
(73,76)\end{array}$ & $\begin{array}{c}380 \\
(47,15)\end{array}$ \\
\hline Associação & $\begin{array}{l}48.387 \\
(19,25)\end{array}$ & $\begin{array}{c}1.546 \\
(12,92)\end{array}$ & $\begin{array}{l}24.766 \\
(17,30)\end{array}$ & $\begin{array}{c}731 \\
(12,31)\end{array}$ & $\begin{array}{l}17.135 \\
(29,75)\end{array}$ & $\begin{array}{c}394 \\
(18,63)\end{array}$ & $\begin{array}{c}9.232 \\
(18,74)\end{array}$ & $\begin{array}{c}454 \\
(19,65)\end{array}$ & $\begin{array}{l}1.115 \\
(4,68)\end{array}$ & $\begin{array}{c}178 \\
(6,74)\end{array}$ & $\begin{array}{c}326 \\
(4,28)\end{array}$ & $\begin{array}{c}55 \\
(6,82)\end{array}$ \\
\hline Fundação & $\begin{array}{l}7.569 \\
(3,01)\end{array}$ & $\begin{array}{c}341 \\
(2,85)\end{array}$ & $\begin{array}{l}2.568 \\
(1,79)\end{array}$ & $\begin{array}{c}23 \\
(0,39)\end{array}$ & $\begin{array}{c}127 \\
(0,22)\end{array}$ & $\begin{array}{c}7 \\
(0,33)\end{array}$ & $\begin{array}{c}25 \\
(0,05)\end{array}$ & $\begin{array}{c}4 \\
(0,17)\end{array}$ & $\begin{array}{c}56 \\
(0,23)\end{array}$ & $\begin{array}{c}4 \\
(0,15)\end{array}$ & $\begin{array}{c}13 \\
(0,17)\end{array}$ & $\begin{array}{c}3 \\
(0,37)\end{array}$ \\
\hline Governo & $\begin{array}{l}2.644 \\
(1,05)\end{array}$ & $\begin{array}{c}36 \\
(0,30)\end{array}$ & $\begin{array}{c}784 \\
(0,55)\end{array}$ & $\begin{array}{c}36 \\
(0,61)\end{array}$ & $\begin{array}{c}334 \\
(0,58)\end{array}$ & $\begin{array}{c}19 \\
(0,90)\end{array}$ & $\begin{array}{c}241 \\
(0,49)\end{array}$ & $\begin{array}{c}8 \\
(0,35)\end{array}$ & $\begin{array}{c}387 \\
(1,62)\end{array}$ & $\begin{array}{c}26 \\
(0,98)\end{array}$ & $\begin{array}{c}184 \\
(2,42)\end{array}$ & $\begin{array}{c}23 \\
(2,85)\end{array}$ \\
\hline $\begin{array}{l}\text { Instituto de } \\
\text { Pesquisa }\end{array}$ & $\begin{array}{l}1.703 \\
(0,68)\end{array}$ & $\begin{array}{c}102 \\
(0,85)\end{array}$ & $\begin{array}{l}4.444 \\
(3,10)\end{array}$ & $\begin{array}{c}136 \\
(2,29)\end{array}$ & $\begin{array}{l}1.506 \\
(2,61)\end{array}$ & $\begin{array}{c}81 \\
(3,83)\end{array}$ & $\begin{array}{l}1.182 \\
(2,40)\end{array}$ & $\begin{array}{c}106 \\
(4,59)\end{array}$ & $\begin{array}{c}473 \\
(1,98)\end{array}$ & $\begin{array}{c}40 \\
(1,52)\end{array}$ & $\begin{array}{c}512 \\
(6,73)\end{array}$ & $\begin{array}{c}24 \\
(2,98)\end{array}$ \\
\hline Outras & $\begin{array}{l}3.068 \\
(1,22) \\
\end{array}$ & $\begin{array}{c}198 \\
(1,65) \\
\end{array}$ & $\begin{array}{l}1.963 \\
(1,37) \\
\end{array}$ & $\begin{array}{c}102 \\
(1,72) \\
\end{array}$ & $\begin{array}{c}115 \\
(0,20) \\
\end{array}$ & $\begin{array}{c}19 \\
(0,90) \\
\end{array}$ & $\begin{array}{c}248 \\
(0,51) \\
\end{array}$ & $\begin{array}{c}22 \\
(0,95) \\
\end{array}$ & $\begin{array}{c}758 \\
(3,18) \\
\end{array}$ & $\begin{array}{c}42 \\
(1,59) \\
\end{array}$ & $\begin{array}{c}88 \\
(1,16) \\
\end{array}$ & $\begin{array}{c}22 \\
(2,73) \\
\end{array}$ \\
\hline Total & $\begin{array}{l}251.312 \\
(100,00)\end{array}$ & $\begin{array}{c}11.965 \\
(100,00) \\
\end{array}$ & $\begin{array}{l}143.135 \\
(100,00) \\
\end{array}$ & $\begin{array}{c}5.936 \\
(100,00)\end{array}$ & $\begin{array}{l}57.604 \\
(100,00)\end{array}$ & $\begin{array}{c}2.115 \\
(100,00)\end{array}$ & $\begin{array}{c}49.276 \\
(100,00)\end{array}$ & $\begin{array}{c}2.310 \\
(100,00)\end{array}$ & $\begin{array}{c}23.846 \\
(100,00)\end{array}$ & $\begin{array}{c}2.640 \\
(100,00)\end{array}$ & $\begin{array}{c}7.611 \\
(100,00)\end{array}$ & $\begin{array}{c}806 \\
(100,00) \\
\end{array}$ \\
\hline
\end{tabular}

Fonte: Elaborado pelas autoras (2020).

Nota: A: Artigos; P: Periódicos.

Os grupos com maior participação na WoS - Elsevier, Springer, Wiley, Taylor \& Francis e Sage Group - editam $72,97 \%$ dos títulos publicados por editores comerciais, respectivamente 20,40\%, 20,37\%, 14,09\%, 13,06\% e 5,05\%, sendo que $76,97 \%$ dos artigos são publicados por essas empresas, nesta ordem: 37,87\%, 19,72\%, 11,68\%, 6,01\% e $1,76 \%$. Nessas empresas, exceto na Elsevier, o percentual de artigos é ligeiramente inferior ao de títulos. As empresas Elsevier, Springer, Wiley, Taylor \& Francis ocupam os primeiros lugares do ranking de artigos. Os publishers em Acesso Aberto Public Library Science (PloS) e Hindawi ocupam a quinta e a sexta posições, e a Sage Group ocupa a sétima. Esses dados indicam que mesmo entre publishers comerciais existe um núcleo mais influente. As dez empresas no topo do ranking (nesta ordem: Elsevier, Springer, Wiley, Taylor \& Francis, PLoS, Hindawi, Sage Group, Wolters Kluwer, Magnolia e MDPI) são publicadoras de 85,13\% dos artigos e 77,89\% dos periódicos.

Entre esses publishers, dois apresentam um número reduzido de títulos, enquanto os demais apresentam de dezenas a milhares, dado que revela a fragmentação dos grupos editoriais. A concentração de artigos por título é mais elevada em grupos que editam menor número de periódicos, como Magnolia Press e PLoS, editores de mega journals. Elsevier, Springer, Wiley e Taylor \& Francis possuem médias de artigo por títulos menor e maior volume de títulos (Tabela 2).

O mega journal PLoS One é o periódico que concentra o maior número de artigos e o maior investimento em pagamento de Article Processing Charge (APC) (Tabela 2). A estimativa parcial do investimento regional em publicação, apenas para os títulos ranqueados, é de US\$18.950.401,5; mais de US\$6 milhões ao ano - valor superior ao destinado à publicação de periódicos pela Coordenação de Aperfeiçoamento de Pessoal de Nível Superior (Capes) e pelo Conselho Nacional de Desenvolvimento Científico e Tecnológico (CNPq) no Brasil ${ }^{4}$, de R\$4 milhões (Coordenação de Aperfeiçoamento de Pessoal de Nível Superior, 2018); aproximadamente US\$735.0005. O contraste dessa comparação mostra a existência de uma sobreposição de investimentos para publicação e acesso

\footnotetext{
${ }^{4}$ Referente à Chamada CNPq/Capes no 18/2018 do Programa Editorial, como parte de uma política de desenvolvimento científico e tecnológico, com o objetivo de apoiar a editoração e publicação de periódicos científicos brasileiros em todos os campos de conhecimento (Coordenação de Aperfeiçoamento de Pessoal de Nível Superior, 2018). Valor reduzido em 75\% para a chamada 2019, para a qual não houve dotação de recurso orçamentário pela Capes (Rocha, 2019).

5 Estimado pela cotação do dólar a R\$5,45 em janeiro de 2021.
} 
Tabela 2 - Ranking de periódicos com maior volume de artigos (superior a 300) por publishers comerciais, associações e universidades.

1 de 2

\begin{tabular}{|c|c|c|c|c|c|c|c|}
\hline Título & Comercial & País & Acesso & $\mathrm{FI}$ & Artigos (N) & APC (US\$) & Total APC (US\$) \\
\hline PLoS One & PLOS & EUA & $\mathrm{OA}$ & 2.740 & 4.566 & 1.695 & $7.739 .370,0$ \\
\hline Zootaxa & Magnolia & NZL & $\mathrm{OA}$ & 0.955 & 1.556 & 200 & $311.200,0$ \\
\hline Astronomy \& Astrophysics & EDP Sciences & FRA & $\mathrm{OA}$ & 5.636 & 1.240 & $3.647,41$ & $4.522 .788,4$ \\
\hline Scientific Reports & Springer & GBR & $\mathrm{OA}$ & 3.998 & 835 & $2.054,71$ & $1.715 .682,85$ \\
\hline Phytotaxa & Magnolia & $\mathrm{NZL}$ & $\mathrm{OA}$ & 1.007 & 693 & 200 & $138.600,0$ \\
\hline Nutrición Hospitalaria & Aula Medica & ESP & $\mathrm{OA}$ & 0.888 & 575 & 547,11 & $314.588,25$ \\
\hline PLoS Neglected Tropical Diseases & PLOS & EUA & $\mathrm{OA}$ & 3.885 & 534 & 2.350 & $1.254 .900,0$ \\
\hline Biomed Research International & Hindawi & EUA & $\mathrm{OA}$ & 2.276 & 530 & 2400 & $1.272 .000,0$ \\
\hline Food Chemistry & Elsevier & GBR & Subs & 6.306 & 507 & - & - \\
\hline Journal of high energy physics & Springer & EUA & $\mathrm{OA}$ & 5.875 & 441 & 0 & 0 \\
\hline Molecules & MDPI & $\mathrm{CHE}$ & $\mathrm{OA}$ & 3.267 & 400 & $2.247,73$ & $899.092,0$ \\
\hline LWT-Food Science and Technology & Elsevier & NLD & Subs & 4.006 & 394 & - & - \\
\hline Physica A-Statistical Mechanics and its Applications & Elsevier & NLD & Subs & 2.924 & 376 & - & - \\
\hline Journal of South American Earth Sciences & Elsevier & GBR & Subs & 1.704 & 361 & - & - \\
\hline Industrial Crops and Products & Elsevier & NLD & Subs & 4.244 & 358 & - & - \\
\hline International Journal of Hydrogen Energy & Elsevier & GBR & Subs & 4.939 & 340 & - & - \\
\hline Science of The Total Environment & Elsevier & NLD & Subs & 6.551 & 336 & - & - \\
\hline Ceramics International & Elsevier & GBR & Subs & 3.830 & 331 & - & - \\
\hline Food Research International & Elsevier & NLD & Subs & 4.972 & 329 & - & - \\
\hline Environmental Science and Pollution Research & Springer & DEU & Subs & 3.056 & 317 & & \\
\hline Parasites \& Vectors & Springer & GBR & $\mathrm{OA}$ & 2.824 & 302 & 2.590 & $782.180,0$ \\
\hline Physics Letters B & Elsevier & NLD & $\mathrm{OA}$ & 4.384 & 300 & 0 & 0 \\
\hline
\end{tabular}

\begin{tabular}{|c|c|c|c|c|c|c|c|}
\hline \multirow[b]{2}{*}{ Título } & \multirow[b]{2}{*}{ Associação } & \multicolumn{5}{|c|}{ Total estimado (publishers comerciais) } & \multirow{2}{*}{$\begin{array}{c}18.950 .401,5 \\
\text { Total APC (US\$) }\end{array}$} \\
\hline & & País & Acesso & $\mathrm{FI}$ & $\operatorname{Artigos}(\mathrm{N})$ & ) APC (US\$) & \\
\hline IEEE Latin America Transactions & IEEE & EUA & Subs & 0.782 & 1249 & - & - \\
\hline Physical Review D & APS & EUA & Subs & 4.833 & 1221 & - & \\
\hline Ciência \& saúde coletiva & ABRASCO & BRA & $\mathrm{OA}$ & 1.019 & 931 & 18,35 & $17.083,85$ \\
\hline RSC advances & Royal Soc Chemistry & GBR & Subs & 3.119 & 723 & - & - \\
\hline Physical Review E & APS & EUA & Subs & 2.296 & 626 & - & - \\
\hline Physical Review B & APS & EUA & Subs & 3.575 & 624 & - & - \\
\hline Journal of the Brazilian Chemical Society & SBQ & BRA & $\mathrm{OA}$ & 1.399 & 593 & 266,05 & $157.767,65$ \\
\hline Química Nova & SBQ & BRA & $\mathrm{OA}$ & 0.668 & 533 & 174,31 & $92.907,23$ \\
\hline Revista Medica de Chile & Soc Medica Santiago & $\mathrm{CHL}$ & Subs & 0.531 & 524 & - & - \\
\hline Anais da Academia Brasileira de Ciências & $A B C$ & BRA & $\mathrm{OA}$ & 1.28 & 512 & 0 & 0 \\
\hline Physical Review A & APS & EUA & Subs & 2.777 & 494 & - & \\
\hline Revista Brasileira de Ciência do Solo & SBCS & BRA & OA & 1.2 & 411 & 50 & $20.550,0$ \\
\hline Journal of Physical Chemistry C & ACS & EUA & Subs & 4.189 & 402 & - & - \\
\hline Physical Chemistry Chemical Physics & RSC & GBR & Subs & 3.43 & 399 & - & - \\
\hline Arquivos de Neuro-Psiquiatria & ABNEURO & BRA & $\mathrm{OA}$ & 1.008 & 385 & 0 & 0 \\
\hline International Journal of Morphology & Soc Chilena Anatomia & $\mathrm{CHL}$ & $\mathrm{OA}$ & 0.499 & 375 & 0 & 0 \\
\hline Anais Brasileiros de Dermatologia & SBD & BRA & $\mathrm{OA}$ & 1.121 & 365 & 0 & 0 \\
\hline Revista Brasileira de Fruticultura & SBF & BRA & $\mathrm{OA}$ & 0.614 & 362 & 211,01 & $76.385,62$ \\
\hline Revista da Sociedade Brasileira de Medicina Tropical & SBMT & BRA & $\mathrm{OA}$ & 1.339 & 359 & 0 & 0 \\
\hline Engenharia Agrícola & SBEA & ESP & $\mathrm{OA}$ & 0.603 & 345 & 113,76 & $39.247,2$ \\
\hline Physical Review Letters & APS & EUA & Subs & 8.385 & 336 & - & - \\
\hline Interciencia & Assoc Interciencia & VEM & Subs & 0.448 & 329 & - & - \\
\hline Acta Cirúrgica Brasileira & SOBRADPEC & BRA & $\mathrm{OA}$ & 0.974 & 323 & 275,23 & $88.899,29$ \\
\hline Gaceta Medica de Mexico & ANMM & MEX & Subs & 0.581 & 321 & - & - \\
\hline \multirow[t]{2}{*}{ Proceedings of the National Academy of Sciences } & NAS & EUA & Subs & 9.412 & 309 & - & - \\
\hline & & \multicolumn{5}{|c|}{ Total estimado (publishers associações) } & $492.840,84$ \\
\hline
\end{tabular}


Tabela 2 - Ranking de periódicos com maior volume de artigos (superior a 300) por publishers comerciais, associações e universidades.

\begin{tabular}{|c|c|c|c|c|c|c|c|}
\hline Título & Universidade & País & Acesso & $\mathrm{FI}$ & Artigos (N) & APC (US\$) & Total APC (US\$) \\
\hline Monthly Notices of the Royal Astronomical Society & Oxford Univ & GBR & Subs. & 5.357 & 1.305 & - & - \\
\hline Semina: Ciências Agrárias & UEL & BRA & $\mathrm{OA}$ & 0.412 & 1.075 & 73,44 & $78.948,0$ \\
\hline Ciência Rural & UFSM & BRA & $\mathrm{OA}$ & 0.556 & 1.040 & 238,68 & $248.227,2$ \\
\hline Arquivo Brasileiro de Medicina Veterinária e Zootecnia & UFMG & BRA & $\mathrm{OA}$ & 0.279 & 708 & 286,41 & $202.778,28$ \\
\hline Bioscience Journal & UFU & BRA & $\mathrm{OA}$ & 0.268 & 665 & 91,74 & $61.007,1$ \\
\hline Pesquisa Veterinária Brasileira & UNB | UFRRJ & BRA & $\mathrm{OA}$ & 0.440 & 583 & 275,4 & $160.558,2$ \\
\hline Revista Brasileira de Engenharia Agrícola e Ambiental & UFCG & BRA & $\mathrm{OA}$ & 0.654 & 537 & 110,16 & $59.155,92$ \\
\hline Materials Research & UFSCar & BRA & $\mathrm{OA}$ & 1.468 & 475 & 146,88 & $69.768,0$ \\
\hline Revista Mexicana de Biodiversidad & UNAM & MEX & $\mathrm{OA}$ & 0.585 & 464 & 0 & 0 \\
\hline Revista de Biologia Tropical & UCR & CRC & $\mathrm{OA}$ & 0.446 & 438 & 0 & 0 \\
\hline Revista da Escola de Enfermagem da USP & USP & BRA & $\mathrm{OA}$ & 0.798 & 403 & 367,19 & $147.977,57$ \\
\hline Holos & IFRN & BRA & $\mathrm{OA}$ & - & 382 & 0 & 0 \\
\hline Revista Arvore & UFV & BRA & $\mathrm{OA}$ & 0.382 & 355 & 0 & 0 \\
\hline Revista Caatinga & UFERSA & BRA & $\mathrm{OA}$ & 0.713 & 351 & 91,74 & $32.200,74$ \\
\hline Ciência Florestal & UFSM & BRA & $\mathrm{OA}$ & 0.5 & 316 & 55,04 & $17.392,64$ \\
\hline Revista Latino-americana de Enfermagem & USP & BRA & $\mathrm{OA}$ & 1.297 & 315 & 0 & 0 \\
\hline Revista Ciência Agronômica & UFC & BRA & $\mathrm{OA}$ & 0.87 & 314 & 100,92 & $31.688,88$ \\
\hline
\end{tabular}

Fonte: Elaborado pelas autoras (2020).

Nota: Modelo de acesso identificado em WoS. FI 2019. Article Processing Charges (APC) do site do periódico; em dólares - dólar a R\$5,45, euro a US\$1,22, franco suiço a US\$1,12, em janeiro de 2021; por página considera 10 páginas por artigo; somadas taxas de tramitação, submissão (quando existem) e publicação. ABC: Academia Brasileira de Ciências; ABNEURO: Academia Brasileira de Neurologia; ABRASCO: Associação Brasileira de Saúde Coletiva; ACS: American Chemical Society; ANMM: Academia Nacional de Medicina do México; AO: Open Access; APC: Article Processing Charges; APS: American Physical Society; BRA: Brasil; CHE: Suíça; CHL: Chile; CRC: Costa Rica; DEU: Alemanha; ESP: Espanha; EUA: Estados Unidos da América; Fl: Fator de Impacto; FRA: França; GBR: Reino Unido; IEEE: Institute of Electrical and Electronic Engineers; IFRN: Instituto Federal do Rio Grande do Norte; NAS: National Academy of Sciences; MEX: México; NLD: Países Baixos; NZL: Nova Zelândia; PLOS: Public Library Science; RSC: Royal Society of Chemistry; SBCS: Sociedade Brasileira de Ciência do Solo; SBD: Sociedade Brasileira de Dermatologia; SBEA: Associação Brasileira de Engenharia Agrícola; SBF: Sociedade Brasileira de Física; SBMT: Sociedade Brasileira de Medicina Tropical; SBQ: Sociedade Brasileira de Química; SOBRADPEC: Sociedade Brasileira para o Desenvolvimento da Pesquisa em Cirurgia; Subs: Subscrição; UCR: Universidad de Costa Rica; UEL: Universidade Estadual de Londrina; UFC: Universidade Federal do Ceará; UFCG: Universidade Federal de Campina Grande; UFERSA: Universidade Federal Rural do Sem-árido; UFSCar: Universidade Federal de São Carlos; UFMG: Universidade Federal de Minas Gerais; UFRRJ: Universidade Federal Rural do Rio de Janeiro; UFSM: Universidade Federal de Santa Maria; UFV: Universidade Federal Viçosa; UFU: Universidade Federal de Uberlândia; UNAM: Universidad Nacional Autónoma de México; UnB: Universidade de Brasília; USP: Universidade de São Paulo; VEM: Venezuela.

e a tendência a supervalorizar o custeio em publicações comerciais estrangeiras. A atratividade desse mercado se observa na especialização de títulos comerciais em temáticas de países periféricos, como a PLoS Neglected Tropical Diseases.

A adoção da APC não reduz o volume de submissões e publicações, mesmo com o passar do tempo e o aumento do valor das taxas, o que faz com que a hiperinflação da APC seja reconhecida como um fenômeno real (Khoo, 2019). Altas APC estão associadas ao volume maior de artigos (Khoo, 2019), uma demonstração da associação entre o valor da publicação e seu prestígio (Björk; Solomon, 2015). Nesse caso a precificação não está associada aos custos de produção, mas ao prestígio obtido através da publicação, normalmente indicado pelo Fl do periódico.

Cabe destacar que o aumento do número de títulos em Acesso Aberto e do valor das APC não implicam na diminuição dos custos de assinatura. No Brasil, que publica 54,24\% do total de artigos analisados nesta pesquisa (136.321 de 251.312), o Portal de Periódicos da Capes investiu, em 2019, R\$431.397.058,006 (Coordenação de

\footnotetext{
${ }^{6}$ Na última década o investimento anual no Portal de Periódicos da Capes foi de R\$1 19.865.856 em 2010, R\$146.289.830 em 2011, R\$162.612.288 em 2012, R\$173.942.902 em 2013, R\$212.936.982 em 2014, R\$275.937.377 em 2015, R\$357.463.927 em 2016 e R\$402.882.610 em 2017, R\$402.887.527 em 2018 (Coordenação de Aperfeiçoamento de Pessoal de Nível Superior, 2020).
} 
Aperfeiçoamento de Pessoal de Nível Superior, 2020), equivalente a US\$79.155.423,497 - o dobro do valor investido em 2014, mesmo com a crescente adesão a modelos de publicação abertos com cobrança de APC. A convivência dos três modelos de negócios de publicação mostra as limitações da adoção do Acesso Aberto como alternativa aos custos da publicação científica.

Entre escolher publicar em Acesso Aberto pela via platina ou pela via dourada, os pesquisadores preferem o pagamento das APC por considerarem que em alguns campos a via platina não tem avaliação de qualidade suficiente (Khoo, 2019; Walters; Linvill, 2011). A perspectiva indica a permanência das taxas de subscrição que originou a crise dos periódicos, acrescida de uma crise de APC, sobrepondo os dois modelos e ampliando, complexificando, agravando e perpetuando a crise de acesso à informação científica.

A existência de políticas mandatórias para a promoção da Ciência Aberta fomenta ações comerciais para promoção do acesso. Embora esse cenário aponte uma mudança do sistema de comunicação científica, privilegiando a abertura da Ciência, ela acontece baseada no financiamento e foco em oportunidades comerciais (Aspesi et al., 2019; Ellers; Crowter; Harvey, 2017; Fyfe et al., 2017; Siler; Frenken, 2020).

A facilidade de adaptação das empresas que atuam na editoria científica (Aspesi et al., 2019; Fyfe et al., 2017), o uso de estratégia de negócios pautadas em rankings de periódicos baseados no Fl (Guédon, 2019), o controle das métricas de avaliação e a diversificação de sua atuação (Arsenault; Castells, 2008; Aspesi et al., 2019) apontam a necessidade de associação entre diversos atores científicos para promoção de mudanças no sistema de comunicação da Ciência. Credita-se às agências financiadoras, por determinar a alocação dos recursos, papel fundamental na promoção e regulação do Acesso Aberto (Aspesi et al., 2019; European Commission, 2019; Guédon, 2019).

Essas mudanças no cenário editorial apontam a possibilidade de ampliação do oligopólio existente para concentração de recursos para publicação das pesquisas, apoiada na estrutura para alocação de capital simbólico em cartéis (Aspesi et al., 2019; Fyfe et al., 2017).

Elsevier, Taylor \& Francis, Wiley, Springer e Sage Group editam a maioria dos títulos e artigos publicados por publishers comerciais em todos os campos. Das publicações dos campos Ciências da Vida \& Biomedicina, as empresas Elsevier, Taylor \& Francis, Wiley, Springer e Sage Group editam 70,58\% dos periódicos e 77,02\% dos artigos. Do campo Ciências Físicas, $72,84 \%$ dos títulos e $81,86 \%$ dos artigos são editados por elas. Do campo Tecnologia, são $79,35 \%$ dos periódicos e 72,30\% dos artigos. Do campo Ciências Sociais, 81,35\% dos títulos e 85,15\% dos artigos e do campo Artes \& Humanidades são 65,55\% dos títulos e 63,62\% dos artigos (Tabela 3).

Elsevier, grupo que opera com a maior margem de lucro do mercado, 37,00\% (Aspesi et al., 2019), publica o maior número de títulos e artigos. Do campo Tecnologia chega a publicar 46,83\% dos artigos e 31,15\% dos periódicos, constituindo-se no campo de sua maior atuação.

\section{Associações como publishers}

Foram identificadas na WoS 864 associações atuando como publishers da produção latino-americana. As associações publicam o segundo maior volume de artigos (19,25\%) e o terceiro de periódicos (12,92\%), atrás das universidades (14,75\%). Sua atuação é mais expressiva nos campos de Ciências Físicas, Tecnologia e Ciências da Vida \& Biomedicina, dos quais publicam 29,75\%, 18,74\% e 17,30\% dos artigos, respectivamente (Tabela 1).

Entre os editores que concentram o maior número de periódicos estão o Institute of Electrical and Electronic Engineers, IOP Publishing e American Chemical Society, editores de 122, 56 e 50 periódicos e publicadores de 3.223, 3.453 e 2.850 artigos, respectivamente. American Chemical Society, Elsevier, Wiley, Springer e Taylor \& Francis são

7 Estimado pela cotação do dólar a R\$5,45 em janeiro de 2021. 
Tabela 3 - Artigos e periódicos por grupos editoriais comerciais e campo do conhecimento.

\begin{tabular}{|c|c|c|c|c|c|c|c|c|c|c|}
\hline \multirow{3}{*}{ Grupo } & \multirow{2}{*}{\multicolumn{2}{|c|}{$\begin{array}{c}\text { Ciências da Vida \& } \\
\text { Biomedicina }\end{array}$}} & \multirow{2}{*}{\multicolumn{2}{|c|}{$\begin{array}{c}\text { Ciências } \\
\text { Físicas } \\
\mathrm{N}(\%)\end{array}$}} & \multicolumn{2}{|c|}{ Tecnologia } & \multicolumn{2}{|c|}{$\begin{array}{l}\text { Ciências } \\
\text { Sociais }\end{array}$} & \multirow{2}{*}{\multicolumn{2}{|c|}{$\begin{array}{c}\text { Artes \& } \\
\text { Humanidades } \\
N(\%)\end{array}$}} \\
\hline & & & & & \multicolumn{2}{|c|}{$N(\%)$} & \multicolumn{2}{|c|}{$N(\%)$} & & \\
\hline & A & $P$ & A & $P$ & A & $P$ & A & $P$ & A & $P$ \\
\hline Elsevier & $\begin{array}{l}30.926 \\
(35,46)\end{array}$ & $\begin{array}{c}986 \\
(22,19)\end{array}$ & $\begin{array}{l}16.192 \\
(46,96)\end{array}$ & $\begin{array}{c}330 \\
(22,81)\end{array}$ & $\begin{array}{l}16.070 \\
(46,83)\end{array}$ & $\begin{array}{c}492 \\
(31,95)\end{array}$ & $\begin{array}{c}2.385 \\
(29,67)\end{array}$ & $\begin{array}{c}241 \\
(14,64)\end{array}$ & $\begin{array}{c}37 \\
(4,23)\end{array}$ & $\begin{array}{c}8 \\
(2,68)\end{array}$ \\
\hline Springer & $\begin{array}{l}17.397 \\
(19,95)\end{array}$ & $\begin{array}{c}870 \\
(19,58)\end{array}$ & $\begin{array}{c}6.700 \\
(19,43)\end{array}$ & $\begin{array}{c}363 \\
(25,09)\end{array}$ & $\begin{array}{c}4.520 \\
(13,17)\end{array}$ & $\begin{array}{c}343 \\
(22,27)\end{array}$ & $\begin{array}{c}1.119 \\
(13,92)\end{array}$ & $\begin{array}{c}200 \\
(12,15)\end{array}$ & $\begin{array}{c}173 \\
(19,79)\end{array}$ & $\begin{array}{c}41 \\
(13,71)\end{array}$ \\
\hline Wiley & $\begin{array}{l}12.436 \\
(14,33)\end{array}$ & $\begin{array}{l}12.436 \\
(14,33)\end{array}$ & $\begin{array}{l}3.293 \\
(9,55)\end{array}$ & $\begin{array}{c}187 \\
(12,92)\end{array}$ & $\begin{array}{l}1.969 \\
(5,74)\end{array}$ & $\begin{array}{c}141 \\
(9,16)\end{array}$ & $\begin{array}{c}1.237 \\
(15,39)\end{array}$ & $\begin{array}{c}291 \\
(17,68)\end{array}$ & $\begin{array}{c}59 \\
(6,75)\end{array}$ & $\begin{array}{c}32 \\
(10,70)\end{array}$ \\
\hline Taylor \& Francis & $\begin{array}{l}4.971 \\
(5,70)\end{array}$ & $\begin{array}{c}425 \\
(9,57)\end{array}$ & $\begin{array}{l}1.877 \\
(5,44)\end{array}$ & $\begin{array}{c}154 \\
(10,64)\end{array}$ & $\begin{array}{l}1.809 \\
(5,27)\end{array}$ & $\begin{array}{c}185 \\
(12,01)\end{array}$ & $\begin{array}{c}1.385 \\
(17,23)\end{array}$ & $\begin{array}{c}410 \\
(24,91)\end{array}$ & $\begin{array}{c}227 \\
(25,97)\end{array}$ & $\begin{array}{c}95 \\
(31,77)\end{array}$ \\
\hline Sage Group & $\begin{array}{l}1.385 \\
(1,59)\end{array}$ & $\begin{array}{c}169 \\
(3,80)\end{array}$ & $\begin{array}{c}160 \\
(0,46)\end{array}$ & $\begin{array}{c}20 \\
(1,38)\end{array}$ & $\begin{array}{c}441 \\
(1,29)\end{array}$ & $\begin{array}{c}61 \\
(3,96)\end{array}$ & $\begin{array}{c}719 \\
(8,94)\end{array}$ & $\begin{array}{c}197 \\
(11,97)\end{array}$ & $\begin{array}{c}60 \\
(6,86)\end{array}$ & $\begin{array}{c}20 \\
(6,69)\end{array}$ \\
\hline Parcial & $\begin{array}{l}67.176 \\
(77,02)\end{array}$ & $\begin{array}{c}3.136 \\
(70,58)\end{array}$ & $\begin{array}{l}28.222 \\
(81,86)\end{array}$ & $\begin{array}{c}1.054 \\
(72,84)\end{array}$ & $\begin{array}{l}24.809 \\
(72,30)\end{array}$ & $\begin{array}{c}1.222 \\
(79,35)\end{array}$ & $\begin{array}{c}6.845 \\
(85,15)\end{array}$ & $\begin{array}{c}1.339 \\
(81,35)\end{array}$ & $\begin{array}{c}556 \\
(63,61)\end{array}$ & $\begin{array}{c}196 \\
(65,55)\end{array}$ \\
\hline Outros editores & $\begin{array}{l}20.042 \\
(22,98)\end{array}$ & $\begin{array}{c}1.307 \\
(29,42)\end{array}$ & $\begin{array}{c}6.256 \\
(18,44)\end{array}$ & $\begin{array}{c}393 \\
(27,16)\end{array}$ & $\begin{array}{c}9.505 \\
(27,70)\end{array}$ & $\begin{array}{c}318 \\
(20,65)\end{array}$ & $\begin{array}{c}1.194 \\
(14,85)\end{array}$ & $\begin{array}{c}307 \\
(18,65)\end{array}$ & $\begin{array}{c}318 \\
(36,38)\end{array}$ & $\begin{array}{c}103 \\
(34,45)\end{array}$ \\
\hline Total & $\begin{array}{c}87.218 \\
(100,00)\end{array}$ & $\begin{array}{c}4.443 \\
(100,00)\end{array}$ & $\begin{array}{c}34.478 \\
(100,00)\end{array}$ & $\begin{array}{c}1.417 \\
(100,00)\end{array}$ & $\begin{array}{c}34.314 \\
(100,00)\end{array}$ & $\begin{array}{c}1.540 \\
(100,00)\end{array}$ & $\begin{array}{c}8.039 \\
(100,00)\end{array}$ & $\begin{array}{c}1.646 \\
(100,00)\end{array}$ & $\begin{array}{c}874 \\
(100,00)\end{array}$ & $\begin{array}{c}299 \\
(100,00)\end{array}$ \\
\hline
\end{tabular}

Fonte: Elaborado pelas autoras (2020).

Nota: A: Artigos; P: Periódicos.

identificados como os cinco maiores publishers nas áreas de Ciência Médicas e Naturais na WoS (Lariviére; Haustein; Mongeo, 2015). O maior volume de artigos publicado (3.589) foi identificado nos 14 títulos editados pela American Physical Society (Tabela 4).

Das 864 associações identificadas, 116 são latino-americanas (13,43\%), editoras de 120 periódicos (7,76\%) e 17.282 artigos (35,72\%). Destacam-se as Sociedade Brasileira de Química (1.126), Associação Brasileira de Saúde Coletiva (931), Academia Brasileira de Ciências (512), Sociedade Brasileira de Ciência do Solo (411) e a chilena Sociedad Médica de Santiago (524), que publicaram 3.504 artigos em 6 periódicos. Comparados aos valores de APC praticados por editores comerciais, cobram taxas de publicação menores (Tabela 2). Embora editoras de um baixo volume de periódicos indexados na WoS, as associações, juntamente com as universidades, são apontadas como publishers com atuação mais relevante na região se comparadas aos comerciais (Rodrigues; Abadal, 2014).

\section{Publishers universitários}

Foram identificadas 668 universidades atuando como publishers da produção latino-americana na WoS. Dessas, 264 são latino-americanas, editoras de 32,18\% dos títulos e 74,61\% dos artigos publicados por universidades (Tabela 5), revelando a importância dessas instituições para publicação e visibilidade da produção científica.

Em todos os campos o maior volume de títulos publicados por universidades é estrangeiro $(67,82 \%)$ são 836 (69,84\%) e 9.905 artigos (84,82\%) publicados em três países: Inglaterra, Espanha e Estados Unidos da América, que juntos publicam $47,36 \%$ dos periódicos e $21,53 \%$ dos artigos editados por universidades. Em quatro dos cinco campos, exceto Ciências Físicas, a maioria dos artigos é publicada por universidades latino-americanas; superior a 75,00\% dos artigos publicados por universidades. Em Artes \& Humanidades, as universidades latino-americanas, publicam a maioria dos artigos enquanto editam a minoria dos títulos. O mesmo ocorre nos campos Ciências da Vida e Biomedicina e Ciências Sociais. Esses dados coincidem com outros estudos sobre a escassa indexação de periódicos editados por universidades na WoS, sendo que o campo de maior expressão é Artes \& Humanidades, possui uma de cada três revistas indexadas na base (Repiso, 2019). 
Tabela 4 - Artigos e periódicos publicados por Associações por campo do conhecimento.

\begin{tabular}{|c|c|c|c|c|c|c|c|c|c|c|c|c|}
\hline \multirow[t]{3}{*}{ Associações } & \multicolumn{2}{|c|}{ América Latina } & \multicolumn{2}{|c|}{$\begin{array}{c}\text { Ciências } \\
\text { da Vida \& } \\
\text { Biomedicina }\end{array}$} & \multicolumn{2}{|c|}{ Ciências Físicas } & \multicolumn{2}{|c|}{ Tecnologia } & \multicolumn{2}{|c|}{ Ciências Sociais } & \multicolumn{2}{|c|}{$\begin{array}{c}\text { Artes \& } \\
\text { Humanidades }\end{array}$} \\
\hline & \multicolumn{2}{|c|}{$N(\%)$} & \multicolumn{2}{|c|}{$N(\%)$} & \multicolumn{2}{|c|}{$\mathrm{N}(\%)$} & \multicolumn{2}{|c|}{$N(\%)$} & \multicolumn{2}{|c|}{$N(\%)$} & \multicolumn{2}{|c|}{$N(\%)$} \\
\hline & A & $P$ & A & P & A & P & A & P & A & P & A & $P$ \\
\hline \multirow[t]{2}{*}{ American Physical Society } & 3.589 & 14 & 292 & 12 & 3.582 & 12 & - & - & 7 & 2 & - & - \\
\hline & $(7,42)$ & $(0,91)$ & $(1,18)$ & $(1,64)$ & $(20,90)$ & $(3,05)$ & - & - & $(0,63)$ & $(1,12)$ & - & - \\
\hline \multirow[t]{2}{*}{ Institute of Physics Publishing } & 3.453 & 56 & 139 & 10 & 3.112 & 40 & 559 & 22 & 70 & 1 & - & - \\
\hline & $(7,14)$ & $(3,62)$ & $(0,56)$ & $(1,37)$ & $(18,16)$ & $(10,15)$ & $(6,06)$ & $(4,85)$ & $(6,28)$ & $(0,56)$ & - & - \\
\hline Institute of Electrical Electronics & 3.223 & 122 & 139 & 20 & 390 & 20 & 3.182 & 121 & 7 & 2 & - & \\
\hline Engineers & $(6,66)$ & $(7,89)$ & $(0,56)$ & $(2,74)$ & $(2,28)$ & $(95,08)$ & $(34,47)$ & $(26,65)$ & $(0,63)$ & $(1,12)$ & - & - \\
\hline \multirow[t]{2}{*}{ American Chemical Society } & 2.850 & 50 & 887 & 28 & 1.714 & 32 & 1444 & 19 & 42 & 1 & - & - \\
\hline & $(5,89)$ & $(3,23)$ & $(3,58)$ & $(3,83)$ & $(10.00)$ & $(8,12)$ & $(15,64)$ & $(4,19)$ & $(3,77)$ & $(0,56)$ & - & - \\
\hline \multirow[t]{2}{*}{ Royal Society of Chemistry } & 2.677 & 36 & 637 & 19 & 2.358 & 26 & 657 & 14 & 7 & 1 & - & - \\
\hline & $(5,53)$ & $(2,33)$ & $(2,57)$ & $(2,60)$ & $(13,76)$ & $(6,60)$ & $(7,12)$ & $(3,08)$ & $(0,63)$ & $(0,56)$ & - & - \\
\hline \multirow[t]{2}{*}{ Sociedade Brasileira Química } & 1.126 & 2 & - & - & 1.126 & 2 & - & - & - & - & - & - \\
\hline & $(2,33)$ & $(0,13)$ & - & & $(6,57)$ & $(0,51)$ & - & - & - & & - & \\
\hline Associação Brasileira de Saúde & 931 & 1 & 931 & 1 & - & - & - & - & - & - & - & - \\
\hline Coletiva & $(1,92$ & $(0,06)$ & $(3,76)$ & $(0,14)$ & - & - & - & - & - & - & - & - \\
\hline American Society for & 700 & 12 & 700 & 12 & - & - & - & - & - & - & - & - \\
\hline Microbiology & $(1,45)$ & $(0,78)$ & $(2,83)$ & $(1,64)$ & - & - & - & - & - & & - & - \\
\hline \multirow[t]{2}{*}{ Optical Society of America } & 644 & 11 & 29 & 2 & 638 & 11 & 46 & 2 & - & - & - & - \\
\hline & $(1,33)$ & $(0,71)$ & $(0,12)$ & $(0,27)$ & $(3,72)$ & $(2,79)$ & $(0,50)$ & $(0,44)$ & & & - & \\
\hline \multirow[t]{2}{*}{ American Geophysical Union } & 565 & 16 & 113 & 5 & 561 & 16 & 7 & 1 & - & - & - & - \\
\hline & $(1,17)$ & $(1,03)$ & $(0,46)$ & $(0,68)$ & $(3,27)$ & $(4,06)$ & $(0,08)$ & $(0,22)$ & - & - & - & - \\
\hline \multirow[t]{2}{*}{ Sociedad Medica Santiago } & 524 & 1 & 524 & 1 & - & - & - & - & - & - & - & - \\
\hline & $(1,08)$ & $(0,06)$ & $(2,12)$ & $(0,14)$ & & & - & - & & & - & \\
\hline \multirow[t]{2}{*}{ Academia Brasileira de Ciências } & 512 & 1 & - & - & - & - & 512 & 1 & - & - & - & - \\
\hline & $(1,06)$ & $(0,06)$ & - & & & & $(5,55)$ & $(0,22)$ & & & - & \\
\hline \multirow[t]{2}{*}{ Parcial } & 20.794 & 322 & 4.391 & 110 & 13.481 & 159 & 6407 & 180 & 133 & 7 & - & - \\
\hline & $(42,97)$ & $(20,83)$ & $(17,73)$ & $(15,05)$ & $(78,68)$ & $(40,36)$ & $(69,40)$ & $(39,65)$ & $(11,93)$ & $(3,93)$ & - & - \\
\hline \multirow[t]{2}{*}{ Outras Associações } & 27.593 & 1224 & 20.375 & 621 & 3654 & 235 & 2825 & 274 & 982 & 171 & 326 & 55 \\
\hline & $(57,03)$ & $(79,17)$ & $(82,27)$ & $(84,95)$ & $(21,32)$ & $(59,64)$ & $(30,60)$ & $(60,35)$ & $(88,07)$ & $(96,07)$ & $(100,00)$ & $(100,00)$ \\
\hline \multirow{2}{*}{ Total } & 48.387 & 1.546 & 24.766 & 731 & 17.135 & 394 & 9.232 & 454 & 1.115 & 178 & 326 & 55 \\
\hline & $(100,00)$ & $(100,00)$ & $(100,00)$ & $(100,00)$ & $(100,00)$ & $(100,00)$ & $(100,00)$ & $(100,00)$ & $(100,00)$ & $(100,00)$ & $(100,00)$ & $(100,00)$ \\
\hline
\end{tabular}

Fonte: Elaborado pelas autoras (2020).

Nota: A: Artigos; P: Periódicos.

O Brasil apresenta o maior número de universidades, assim como o maior número de artigos e de títulos em todos os campos, exceto Tecnologia. As universidades brasileiras são mais especializadas na publicação de títulos de Ciências Sociais (99), Ciências da Vida \& Biomedicina (51) e Artes \& Humanidades (49). Na Colômbia, México, Argentina e Venezuela são as Ciências Sociais. No Chile são as Ciências Sociais e Artes \& Humanidades (Tabela 5). Esses dados reiteram a atuação das universidades como publicadoras de periódicos nos campos nos quais os publishers comerciais são menos atuantes (Repiso; Orduña-Malea; Aguaded, 2019).

Os títulos editados por universidades são minoria na WoS, tendendo à concentração em um grupo de instituições anglo-americanas das quais destacam-se Oxford Universitye Cambridge (Repiso; Orduña-Malea; Aguaded, 2019). No ranking de volume de artigos sobressaem as instituições latinas, com destaque para as universidades públicas brasileiras (12 das 18). A primeira posição é ocupada pela Oxford University e a terceira pela Cambridge University, editoras de 225 e 165 periódicos que publicaram 4.580 e 1.941 artigos, respectivamente. Em relação a essas instituições, convém destacar a natureza comercial de sua atuação, inclusive editorial - o que as diferenciam 
Tabela 5 - Artigos e periódicos publicados por Universidades por país e campo do conhecimento.

\begin{tabular}{|c|c|c|c|c|c|c|c|c|c|c|c|c|c|}
\hline \multirow{3}{*}{ País } & \multicolumn{3}{|c|}{ Total } & \multicolumn{2}{|c|}{$\begin{array}{l}\text { Ciências da Vida } \\
\text { \& Biomedicina }\end{array}$} & \multicolumn{2}{|c|}{ Ciências Físicas } & \multicolumn{2}{|c|}{ Tecnologia } & \multicolumn{2}{|c|}{ Ciências Sociais } & \multicolumn{2}{|c|}{$\begin{array}{c}\text { Artes \& } \\
\text { Humanidades }\end{array}$} \\
\hline & \multicolumn{3}{|c|}{$N(\%)$} & \multicolumn{2}{|c|}{$\mathrm{N}(\%)$} & \multicolumn{2}{|c|}{$\mathrm{N}(\%)$} & \multicolumn{2}{|c|}{$N(\%)$} & \multicolumn{2}{|c|}{$\mathrm{N}(\%)$} & \multicolumn{2}{|c|}{$N(\%)$} \\
\hline & $U$ & $A$ & $P$ & A & $P$ & A & $P$ & A & $P$ & A & $P$ & $A$ & $P$ \\
\hline \multirow[t]{2}{*}{ Brasil } & 104 & 20.368 & 214 & 11.585 & 51 & 219 & 6 & 1.697 & 12 & 4.781 & 99 & 2.441 & 49 \\
\hline & $(15,57)$ & $(44,28)$ & $(12,12)$ & $(54,16)$ & $(10,97)$ & $(5,60)$ & $(3,37)$ & $(42,07)$ & $(6,82)$ & $(36,73)$ & $(14,06)$ & $(43,48)$ & $(12,89)$ \\
\hline \multirow[t]{2}{*}{ Colômbia } & 56 & 3.999 & 127 & 709 & 14 & 42 & 4 & 463 & 15 & 2.206 & 76 & 575 & 17 \\
\hline & $(8,38)$ & $(8,69)$ & $(7,20)$ & $(3,31)$ & $(3,01)$ & $(1,07)$ & $(2,25)$ & $(11,48)$ & $(8,52)$ & $(16,95)$ & $(10,8)$ & $(10,24)$ & $(4,47)$ \\
\hline \multirow[t]{2}{*}{ México } & 23 & 2.717 & 47 & 1.019 & 10 & 508 & 6 & 371 & 5 & 928 & 23 & 112 & 5 \\
\hline & $(3,44)$ & $(5,91)$ & $(2,66)$ & $(4,76)$ & $(2,15)$ & $(13,00)$ & $(3,37)$ & $(9,20)$ & $(2,84)$ & $(7,13)$ & $(3,27)$ & $(2,00)$ & $(1,32)$ \\
\hline \multirow[t]{2}{*}{ Chile } & 22 & 3.213 & 60 & 1.505 & 14 & 274 & 3 & 144 & 4 & 829 & 23 & 875 & 22 \\
\hline & $(3,29)$ & $(6,99)$ & $(3,40)$ & $(7,04)$ & $(3,01)$ & $(7,01)$ & $(1,69)$ & $(3,57)$ & $(2,27)$ & $(6,37)$ & $(3,27)$ & $(15,59)$ & $(5,79)$ \\
\hline \multirow[t]{2}{*}{ Argentina } & 15 & 1.010 & 36 & 353 & 6 & - & - & 111 & 3 & 578 & 23 & 85 & 5 \\
\hline & $(2,25)$ & $(2,20)$ & $(2,04)$ & $(1,65)$ & $(1,29)$ & - & - & $(2,75)$ & $(1,70)$ & $(4,44)$ & $(3,27)$ & $(1,51)$ & $(1,32)$ \\
\hline \multirow[t]{2}{*}{ Venezuela } & 11 & 981 & 33 & 446 & 9 & 6 & 1 & 54 & 2 & 429 & 17 & 59 & 5 \\
\hline & $(1,65)$ & $(2,13)$ & $(1,87)$ & $(2,08)$ & $(1,94)$ & $(0,15)$ & $(0,56)$ & $(1,34)$ & $(1,14)$ & $(3,30)$ & $(2,41)$ & $(1,05)$ & $(1,32)$ \\
\hline \multirow[t]{2}{*}{ Equador } & 8 & 252 & 10 & 5 & 1 & - & - & 49 & 1 & 158 & 6 & 40 & 2 \\
\hline & $(1,20)$ & $(0,55)$ & $(0,57)$ & $(0,02)$ & $(0,22)$ & - & - & $(1,21)$ & $(0,57)$ & $(1,21)$ & $(0,85)$ & $(0,71)$ & $(0,53)$ \\
\hline \multirow[t]{2}{*}{ Cuba } & 7 & 557 & 8 & 23 & 1 & - & - & 24 & 1 & 510 & 6 & - & - \\
\hline & $(1,05)$ & $(1,21)$ & $(0,45)$ & $(0,11)$ & $(0,22)$ & - & - & $(0,59)$ & $(0,57)$ & $(3,92)$ & $(0,85)$ & & - \\
\hline \multirow[t]{2}{*}{ Peru } & 6 & 193 & 8 & 29 & 1 & - & - & 48 & 1 & 116 & 6 & - & - \\
\hline & $(0,90)$ & $(0,42)$ & $(0,45)$ & $(0,14)$ & $(0,22)$ & - & - & $(1,19)$ & $(0,57)$ & $(0,89)$ & $(0,85)$ & - & - \\
\hline \multirow[t]{2}{*}{ Costa Rica } & 5 & 878 & 18 & 487 & 5 & - & - & 102 & 3 & 265 & 9 & 24 & 1 \\
\hline & $(0,75)$ & $(1,91)$ & $(1,02)$ & $(2,28)$ & $(1,08)$ & - & - & $(2,53)$ & $(1,70)$ & $(2,04)$ & $(1,28)$ & $(0,43)$ & $(0,26)$ \\
\hline \multirow[t]{2}{*}{ Uruguai } & 3 & 72 & 3 & - & - & - & - & 11 & 1 & 61 & 2 & - & - \\
\hline & $(0,45)$ & $(0,16)$ & $(0,17)$ & - & - & - & - & $(0,27)$ & $(0,57)$ & $(0,47)$ & $(0,28)$ & & - \\
\hline Nicarágua & 2 & 42 & 2 & - & - & - & - & 4 & 1 & 38 & 1 & - & - \\
\hline & $(0,30)$ & $(0,09)$ & $(0,11)$ & - & - & - & - & $(0,10)$ & $(0,57)$ & $(0,29)$ & $(0,14)$ & - & - \\
\hline El salvador & 1 & 15 & 1 & - & - & - & - & - & - & - & - & 15 & 1 \\
\hline & $(0,15)$ & $(0,03)$ & $(0,06)$ & - & - & - & - & - & - & - & - & $(0,27)$ & $(0,26)$ \\
\hline Paraguai & 1 & 20 & 1 & - & - & & - & - & - & 20 & 1 & - & - \\
\hline & $(0,15)$ & $(0,04)$ & $(0,06)$ & - & - & - & - & - & - & $(0,15)$ & $(0,14)$ & - & - \\
\hline Parcial & 264 & 34.317 & 568 & 16.161 & 112 & 1.049 & 20 & 3.078 & 49 & 10.919 & 292 & 4.226 & 107 \\
\hline & $(39,52)$ & $(74,61)$ & $(32,18)$ & $(75,55)$ & $(24,09)$ & $(26,84)$ & $(11,24)$ & $(76,30)$ & $(27,84)$ & $(83,88)$ & $(41,48)$ & $(75,28)$ & $(28,16)$ \\
\hline Outros Países & 404 & 11.677 & 1.197 & 5.231 & 353 & 2.860 & 158 & 956 & 127 & 2.099 & 412 & 1.388 & 273 \\
\hline & $(60,48)$ & $(25,39)$ & $(67,82)$ & $(24,45)$ & $(75,91)$ & $(73,16)$ & $(88,76)$ & $(23,70)$ & $(72,16)$ & $(16,12)$ & $(58,52)$ & $(24,72)$ & $(71,84)$ \\
\hline & 668 & 45.994 & 1.765 & 21.392 & 465 & 3.909 & 178 & 4.034 & 176 & 13.018 & 704 & 5.614 & 380 \\
\hline & $(100,00)$ & $(100,00)$ & $(100,00)$ & $(100,00)$ & $(100,00)$ & $(100,00)$ & $(100,00)$ & $(100,00)$ & $(100,00)$ & $(100,00)$ & $(100,00)$ & $(100,00)$ & $(100,00)$ \\
\hline
\end{tabular}

Fonte: Elaborado pelas autoras (2020).

Nota: Outros países: Países com maior volume de artigos e periódicos: Inglaterra - A 6.591 (14,33\%), P 405 (22,95\%); Espanha - A 2.212 (4,81\%), P 241 (13,65\%), EUA - A 1.102 (2,39\%), P 190 (10,76\%). A: Artigos; P: Periódicos; U: Universidade.

das universidades latino-americanas. Entre as latino-americanas destacam-se a Universidade de São Paulo (2a), a Universidade Federal de Santa Maria (4a) e a Universidad Nacional Autónoma de México (5a), que publicam, nessa ordem, 2.312, 1.715 e 1.222 artigos em 16, 4 e 15 títulos.

É possível observar que mesmo na América Latina, entre os títulos editados por universidades, tem sido implementada a cobrança de APC (Tabela 2). Comparativamente, o valor é muito menor que o praticado pelos publishers comerciais (Appel; Albagli, 2019). A totalidade dos artigos publicados na Revista da Escola de Enfermagem da Universidade de São Paulo, que possui o maior valor de APC entre os títulos universitários, equivale a US\$147.977,17. O maior valor estimado de APC foi US\$248.227,2 pagos à Ciência Rural para publicação de 1.040 artigos (Tabela 2). 
Embora não apareçam com destaque na WoS entre os títulos editados por universidades por causa da origem anglo-americana dos títulos, as instituições latino-americanas têm expressiva participação no mercado editorial científico. Universidades seguem como as editoras majoritárias dos títulos editados na América Latina, assumindo papel central no esforço editorial científico da região (Björk, 2017; Corera-Álvarez; Molina-Molina, 2016; Rodrigues; Abadal, 2014), e são também as maiores empregadoras de pesquisadores na região (Leta, 2012).

\section{Conclusão}

A análise da produção cientíica latino-americana indexada na WoS permite a identificação do esforço desses países para tornar o conhecimento que produzem mais visível entre as publicações consideradas centrais. É possível perceber na região que existem países que têm obtido maiores visibilidade e volume de trabalhos publicados. A visibilidade fora do centro, ou do universo considerado como global, é obtida com os padrões de comunicação centrais, como a publicação de artigos em títulos estrangeiros, ou pela internacionalização dos títulos nacionais ou regionais.

A história recente da consolidação de mecanismos de comunicação e de construção de instrumentos de avaliação da produção científica regional demonstra o aceite das condições estabelecidas para a participação no grupo de elite, expresso na valorização do Fl e da publicação em inglês, privilegiando títulos estrangeiros. Essa participação evidencia a concordância com a redução de trabalhos escritos nos idiomas nativos e na valorização dos títulos estrangeiros na maioria dos campos do conhecimento.

Entre os países periféricos também os campos considerados periféricos, que tradicionalmente possuem maior restrição em sua representação na base de dados, são os que apresentam menor dependência da estrutura mundial de comunicação da Ciência. A falta de tradição na representação desses campos gerou processos de institucionalização que precisaram definir as formas de comunicação valoradas e que criaram seus veículos de comunicação de modo mais independente da influência do centro. Isso ainda se reflete nos mecanismos de avaliação desses campos que tendem a resistir à exclusividade dos indicadores de bases internacionais como meio de valorar sua produção científica. O oposto ocorre nos campos tradicionalmente internacionalizados e representados na WoS.

Ao reconhecer como ciência global apenas o que é publicado na WoS, a ideia do que é "ciência de qualidade" e onde ela é publicada é reforçada e, como consequência, invisibiliza tudo o que não faz parte desse universo. Desse modo, cria-se entre os membros dos campos científicos a necessidade de fazer parte desse seleto grupo, seja pela publicação nos títulos que já compõem a elite ou pela adoção dos padrões de comunicação desses títulos em uma tentativa de adequar-se ao modelo global de produção científica.

A existência de um "padrão global" de Ciência, baseada nas publicações em títulos indexados em bases internacionais, força os campos a reconhecerem, em seus instrumentos de avaliação da produção científica, os veículos de comunicação já utilizados na região que começam a aparecer entre os títulos mais prestigiados, preterindo os que estão fora do grupo. Esse comportamento transforma em necessidade a produção de comunicações para os títulos que apresentem posição de liderança, estabelecendo dependência dessas publicações para avaliação da produção científica e fortalecendo a atuação de editores comerciais.

Os padrões de produção, publicação e avaliação científicas são moldados pelos títulos mainstream pela perpetuação de noções de internacionalização, visibilidade e qualidade editorial estabelecidas nessas bases de dados. Assim, os parâmetros adotados para participação na elite científica reproduzem os modelos definidos em um contexto restrito e geograficamente limitado, sendo incorporados e reproduzidos nos sistemas de comunicação regionais. 
O predomínio de grupos comerciais na publicação dos títulos indexados na base é evidente; entretanto, sua participação na publicação da produção regional tende a diminuir com a expansão da cobertura da base, evidenciado na proporção dos artigos publicados pelos publishers comerciais - que é inferior ao percentual de títulos. Além disso, sua atuação é maior nos campos tradicionalmente representados na base: Ciências da Vida \& Biomedicina, Ciências Físicas eTecnologia. Nos campos historicamente relegados, as Ciências Humanas e Sociais, as universidades se firmam como publishers.

A concentração de artigos na PloS One e em outros publishers comerciais de Acesso Aberto indica uma tendência do mercado editorial científico: a publicação de mega journals, com milhares de artigos, em Acesso Aberto, pela via dourada e com cobrança de APC. Os grupos editoriais pautam a oferta desses serviços pelo prestígio do título, perpetuando o mecanismo de lucro e a atuação de grandes conglomerados editoriais comerciais.

Em países latino-americanos vigora a edição de títulos abertos subsidiados pela instituição editora, pela via platina, sendo a maioria publicada por universidades e associações científicas. Ainda que tenha sido identificada a cobrança de APC, os valores arrecadados são muito mais baixos do que os praticados pelos publishers comerciais e o modelo de publicação de periódicos na região é diferente dos títulos editados no estrangeiro.

Esses resultados são relevantes para discussões de políticas científicas, pela representação da institucionalização dos campos de pesquisa refletida na existência de veículos de comunicação consolidados e pela análise dos modelos de negócio adotados nos títulos publicados na América Latina.

O sucesso do Open Access em regiões periféricas e as iniciativas mundiais para sua promoção são indícios da capacidade dos países de promover mudanças, inovações e de seus esforços para participar do sistema global de comunicação da Ciência. Entretanto, as tendências de expansão do Open Access promovidas por publishers comerciais e as altas APC cobradas e a manutenção do modelo de assinatura dos periódicos por subscrição refletem a força dos agentes que ocupam posições de poder no sistema de comunicação científica e o sucesso deles em se adaptarem às mudanças que garantem a continuidade da lucratividade de seus negócios e a ampliação dos seus instrumentos de controle da ciência.

\section{Colaboradores}

Ambas as autoras participaram da concepção, análise, redação, revisão e aprovação final do artigo.

\section{Referências}

Appel, A. L.; Albagli, S. The adoption of Article Processing Charges as a business model by Brazilian Open Access journals. Transinformação, v. 31, e180045, 2019. Doi: http:// dx.doi.org/10.1590/2318-0889201931e180045.

Arsenault, A.; Castells, M. The structure and dynamics of global multi-media business networks. International Journal of Communication, v. 2, p. 707-748, 2008.

Aspesi, C. et al. SPARC landscape analysis: the changing academic publishing industry: implications for academic institutions. Washington: SPARC, 2019. Available from: https:// sparcopen.org/our-work/landscape-analysis/. Cited: Jan. 5, 2020.

Björk, B. Journal portals - an important infrastructure for non-commercial scholarly open access publishing. Online Information Review, v. 41, n. 5, p. 644-654, 2017. Doi: https:// doi.org/10.1108/OIR-03-2016-0088.

Björk, B.; Solomon, D. Article processing charges in OA journals: relationship between price and quality. Scientometrics, v. 103, n. 2, p. 373-385, 2015. Doi: https://doi.org/10.1007/s11192015-1556-z.

Coordenação de Aperfeiçoamento de Pessoal de Nível Superior. Edital destina $\mathrm{R} \$ 4$ milhões para incentivar a publicação de periódicos científicos. Capes, Brasília, 13 ago. 2018. Disponível em: http://www1.capes.gov.br/36noticias/8991-edital-destina-r-4-milhoes-para-incentivar-apublicacao-de-periodicos-cientificos. Acesso em: 25 nov. 2019.

Coordenação de Aperfeiçoamento de Pessoal de Nível Superior. Orçamento: evolução em reais. Capes, Brasília, 29 set. 2020. Disponível em: https://www.gov.br/capes/pt-br/ acesso-a-informacao/institucional/orcamento-evolucao-emreais. Acesso em: 4 out. 2020.

Corera-Álvarez, E.; Molina-Molina, S. La edición universitaria de revistas científicas. Revista Interamericana de Bibliotecología, v. 39, n. 3, p. 277-288, 2016. Doi: https://doi.org/10.17533/udea. rib.v39n3a05. 
Ellers, J.; Crowther, T.; Harvey, J. Gold Open Access publishing in mega-journals: developing countries pay the price of western premium academic output. Journal of Scholarly Publishing, $\mathrm{v}$. 49, n. 1, p. 89-102, 2017. Doi: https://doi.org/10.3138/jsp.49.1.

Elsevier. Sobre a solução Scopus. Amsterdã: Elsevier, c2021. Disponível em: https://www.elsevier.com/pt-br/solutions/ scopus. Acesso em: 7 jan. 2020.

European Commission. Future of scholarly publishing and scholarly communication: report of the expert group to the European Commission. Luxembourg: Publications Office of the European Union, 2019.

Fyfe, A. et al. Untangling academic publishing: a history of the relationship between commercial interests, academic prestige and the circulation of research. Zenodo, May 25, 2017. Doi: https://doi.org/10.5281/zenodo.546100.

Gingras, Y. The Transformation of the scientific paper: from knowledge to accounting unit. In: Biagioli, M.; Lippman, A. (ed.). Gaming the metrics: misconduct and manipulation in academic research. Boston: MITPress, 2020. Doi: https://doi. org/10.7551/mitpress/11087.003.0004.

Guédon, J. El acceso abierto y la división entre ciencia "principal"y"periférica". Crítica y Emancipación, v. 3, n. 6, p. 135180, 2011.

Guédon, J. Preface. In: European Commission (ed.). Future of scholarly publishing and scholarly communication: report of the expert group to the European Commission. Luxembourg: Publications Office of the European Union, 2019.

Khoo, S. Y. Article processing charge hyperinflation and price insensitivity: an open access sequel to the serials crisis. Liber Quarterly, v.29, n. 1, p. 1-18, 2019. Doi: http://doi.org/10.18352/ lq.10280.

Lachenmayer, D. Let it flow: the monopolization of academic content providers and how it threatens the democratization of information. The Serials Librarian, v. 75, n. 1-4, p. 1-11, 2019. Doi: https://doi.org/10.1080/0361526x.2018.1556189.

Lariviére, V.; Haustein, S.; Mongeo, P.The oligopoly of academic publishers in the digital era. Plos One, v. 10, n. 6, 2015. Doi: https://doi.org/10.1371/journal.pone.0127502.

Leta, J. Brazilian growth in the mainstream science: the role of human resources and national journals. Journal of Scientometric Research, v. 1, n. 1, p. 44-52, 2012.

McGuigan, G. S.; Russell, R. D. The business of academic publishing: a strategic analysis of the academic journal publishing industry and its impact on the future of scholarly publishing. Electronic Journal of Academic and Special Librarianship, v. 9 n. 3, online, 2008. Available from: http:// southernlibrarianship.icaap.org/content/v09n03/mcguigan_ g01.html. Cited: Oct. 12, 2017.
Monbiot, G. Academic publishers make murdoch look like a socialist. The Guardian, United Kingdom, Aug. 29, 2011. Available from: https://www.theguardian.com/ commentisfree/2011/aug/29/academic-publishersmurdoch-socialist. Cited: Nov. 29, 2019.

Repiso, R. ¿Qué valor tienen las revistas científicas para las universidades que las editan? In: Conferencia Internacional de Revistas Académicas en comunicación, 3., 2019, Barcelona. Anales eletrónicos [...]. Barcelona: Universidad Pompeu Fabra, 2019. Doi: https://doi.org/10.5281/zenodo.2593795.

Repiso, R.; Orduña-Malea, E.; Aguaded, I. Revistas científicas editadas por universidades en Web of Science: características y contribución a la marca universidad. Profesional de la Información, v. 28, n. 4, p. 1-10, 2019.

Rocha, L. Nota de esclarecimento - chamada CNPQ. Associação Brasileira de Editores Científicos, Botucatu, 21 nov. 2019. Disponível em: https://www.abecbrasil.org.br/ novo/2019/11/nota-de-esclarecimento-chamada-cnpq/. Acesso em: 13 jan. 2021.

Rodrigues, R.; Abadal, E. Scientific journals in Brazil and Spain: alternative publisher models. Journal of the American Society for Information Science and Technology, v. 65, n. 10, p. 2145-2151, 2014.

Siler, K.; Frenken, K. The pricing of open access journals: diverse niches and sources of value in academic publishing. Quantitative Science Studies, v. 1, n. 1, p. 28-59, 2020. Doi: https://doi.org/10.1162/qss_a_00016.

Thomson Reuters. Thomson Reuters Announces Definitive Agreement to Sell its Intellectual Property \& Science Business to Onex and Baring Asia for $\$ 3.55$ billion. Thomson Reuters, Toronto, July 11, 2016. Available from: https:// www.thomsonreuters.com/en/press-releases/2016/july/ thomson-reuters-announces-definitive-agreement-to-sellits-intellectual-property-science-business.html. Cited: Jan. 5, 2021.

Vessuri, H.; Guédon, J.; Cetto, A. M. Excellence or quality? impact of the current competition regime on science and scientific publishing in Latin America and its implications for development. Current Sociology, v. 62, e647, 2014. Doi: https:// doi.org/10.1177/0011392113512839.

Walters, W. H.; Linvill, A. C. Characteristics of open access journals in six subject areas. College and Research Libraries, v. 72, n. 4, p. 372-392, 2011. Doi: https://doi.org/10.5860/crl-132.

Zepetnek, S. T.; Jia, J. Electronic journals, prestige, and the economics of academic journal publishing. CLC Web: Comparative Literature and Culture, v. 16, n. 1, p. 1-12, 2014. Doi: https://doi.org/10.7771/1481-4374.2426. 